\title{
KPC: Klebsiella pneumoniae carbapenemasa, principal carbapenemasa en enterobacterias
}

\author{
Alejandra Vera-Leiva, Carla Barría-Loaiza, Sergio Carrasco-Anabalón, Celia Lima, \\ Alejandro Aguayo-Reyes, Mariana Domínguez, Helia Bello-Toledo y Gerardo González-Rocha
}

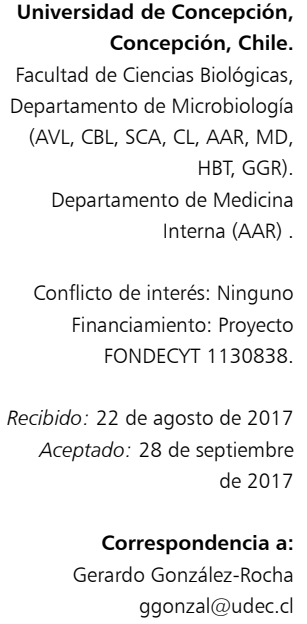

\section{KPC: Klebsiella pneumoniae carbapenemase, main carbapenemase in Enterobacteriaceae}

The dissemination of carbapenemase-producing Enterobacteriaceae is currently considered a serious clinical problem due to the failure in the treatment of infections produced by them. Among the carbapenemases, the enzyme KPC has spread worldwide and has been identified in the main enterobacterial species related with healthcareassociated infections, although Klebsiella pneumoniae is the predominant specie. The $b l a_{\mathrm{KPC}}$ gene is transported, mainly by the transposon Tn4401, detected in various enterobacterial species of different sequence types (ST) and geographical origin. In addition, new genetic platforms that are distinguished, from Tn 4401 because of insertions or deletions of other genes have been described. Plasmids containing the $b l a_{\mathrm{KPC}}$ gene can be conjugative and mobilizable non-conjugative plasmids, and can carry other genetic determinants of resistance. The KPC-producing strains may have different levels of resistance to carbapenems, due to the involvement of additional mechanisms such as different expression levels of porins and efflux pumps associated with the production of extended spectrum $\beta$-lactamases and/or AmpC. However, the carbapenemases, with KPC as the most common enzyme, provide higher levels of resistance.

Keys words: Carbapenemase, genetic platforms, bla $_{\mathrm{KPC}}$, level of resistance.

Palabras clave: Carbapenemasa, plataforma genética, bla $_{\mathrm{KPC}}$, nivel de resistencia.

\section{Introducción}

\section{Carbapenemasas}

Las carbapenemasas representan la familia más versátil de $\beta$-lactamasas, con un amplio espectro de hidrólisis sobre antimicrobianos $\beta$-lactámicos, entre ellos los carbapenémicos. La primera carbapenemasa identificada en enterobacterias fue SME-1 (por "Serratia marcescens enzyme") en Londres en $1982^{1}$ y, posteriormente, en 1984 se describe la enzima IMI-1 (por "imipenem-hydrolyzing $\beta$-lactamase”) en Estados Unidos de América (E.U.A.) ${ }^{2}$. Estas enzimas, al igual que las otras $\beta$-lactamasas, están clasificadas en base a sus propiedades funcionales y moleculares. Así, de acuerdo al esquema de clasificación propuesto por Bush y cols. ${ }^{3}$ las carbapenemasas se encuentran en los grupos 2df, 2f y 3 y, en la clasificación de Ambler, estas enzimas quedan incluidas en las clases A, B y D. Las enzimas clases A y D incluyen a $\beta$-lactamasas que poseen un residuo de serina en su sitio activo, correspondiendo a serin-betalactamasas, mientras que las enzimas de clase B tienen uno o dos iones zinc como cofactor enzimático, denominándose metalo-betalactamasas ${ }^{4}$.

Las carbapenemasas de clase A son las que presentan mayor diversidad y distribución. Se caracterizan por la capacidad para hidrolizar carbapenémicos, cefalosporinas, penicilinas y aztreonam, y han sido identificadas en ente- robacterias y en bacilos gramnegativos no fermentadores. Las principales carbapenemasas de clase A corresponden a: NMC (por "not metallo enzyme carbapenemase"), IMI (por “imipenem-hydrolyzing $\beta$-lactamase”), SME (por "Serratia marcescens enzyme”), GES (por "Guiana extended spectrum”) y KPC (por “Klebsiella pneumoniae carbapenemase" $)^{4,5}$. Las carbapenemasas de clase B se caracterizan por hidrolizar carbapenémicos, con excepción de aztreonam, y su acción es inhibida por el agente quelante EDTA (ácido etilen-diamino-tetra-acético). Las principales metaloenzimas corresponden a: VIM (por "Verona integron-encoded metallo- $\beta$-lactamase), GIM (por "German imipenemase”), SIM (por "Seoul imipenemase”), IMP (por “active on imipenem”) y NDM (por "New Delhi metallo-beta-lactamase"), y han sido descritas en Bacillus cereus, Aeromonas spp., Pseudomonas aeruginosa, Acinetobacter spp. y enterobacterias. Finalmente, las carbapenemasas de clase D, llamadas oxacilinasas, adicionalmente a la hidrólisis de penicilinas, cefalosporinas y carbapenémicos añaden la capacidad de hidrolizar oxacilina y cloxacilina. Estas enzimas han sido identificadas en P. aeruginosa, Acinetobacter spp. y enterobacterias ${ }^{6}$.

En bacilos gramnegativos, la mayoría de las cepas productoras de carbapenamasas corresponden a aislados clínicos de $K$. pneumoniae y Escherichia coli $^{7}$ y las 
carbapenemasas más frecuentemente identificadas son enzimas del tipo KPC, NDM-1, IMP, VIM, OXA-48 (de oxacilinasa) y OXA-181 (Tabla 1$)^{8}$. La amenaza que representan las bacterias productoras de carbapenemasas queda manifestada en los informes entregados por la Organización Mundial de la Salud (OMS). Así, en el año 2014, la OMS informó que las cepas de K. pneumoniae resistentes a carbapenémicos se han diseminado mundialmente y que el principal mecanismo de resistencia es la enzima $\mathrm{KPC}^{9}$. Además, que se ha producido un aumento en la tasa de resistencia a carbapenémicos por sobre $50 \%$, lo cual ha conllevado a un incremento de la mortalidad y morbilidad en pacientes con infecciones por $K$. pneumoniae resistentes a carbapenémicos ${ }^{9}$. Es más, recientemente, a comienzos de 2017, la OMS publicó una lista de patógenos prioritarios que representan la mayor amenaza para la salud humana. Se destaca en el grupo crítico a las bacterias resistentes a los antimicrobianos carbapenémicos como A. baumannii, $P$. aeruginosa y enterobacterias productoras de $\beta$-lactamasas de espectro extendido (BLEE) resistentes a carbapenémicos ${ }^{10}$.

\section{KPC (Klebsiella pneumoniae carbapenemasa)}

El primer reporte de KPC se realizó en el año 1996, en Carolina del Norte, en un aislado de K. pneumoniae ${ }^{11}$. Desde este primer reporte, a la fecha, se han identificado aislados productores de KPC en Europa, Asia, Medio Oriente, América Central y del Sur, África y Oceanía ${ }^{12-15}$.

En América del Sur el primer reporte de KPC-2 fue realizado en Colombia en el año 2006 en K. pneumoniae ${ }^{16}$. Desde esa fecha, la resistencia mediada por KPC se ha diseminado a varias especies de enterobacterias en múltiples hospitales de Colombia ${ }^{15}$. Es así que, el año 2007 se identificó KPC en aislados de $P$. aeruginos $a$, demostrando con este hallazgo diseminación a especies no entéricas ${ }^{17}$.
Por otra parte, en el año 2011, se publicó el primer reporte de un brote de KPC-3 en Colombia y, actualmente, la presencia de cepas productoras de KPC se ha convertido en un problema endémico en este país ${ }^{18}$. En Brasil, Uruguay, Ecuador y Argentina, también se ha reportado la presencia de KPC en aislados de E. coli, Pseudomonas spp., K. pneumoniae, Enterobacter spp., S. marcescens y K. oxytoca ${ }^{12,19-20}$. En Chile, durante los últimos años se registró el aumento en la resistencia o susceptibilidad disminuida a carbapenémicos en enterobacterias, y en marzo de 2012 se realizó el primer hallazgo de una cepa de $K$. pneumoniae productora de KPC, aislada desde un paciente proveniente de Italia ${ }^{21}$. Posteriormente, se informó el primer caso autóctono de KPC, correspondiendo a la presencia de KPC en cepas de K. pneumoniae y $E$. coli aisladas en un hospital de Santiago ${ }^{22}$. Actualmente, se informa un total de 242 aislados productores de carbapenemasas, principalmente de K. pneumoniae, E. cloacae y K. oxytoca, con KPC como carbapenemasa predominante, seguida de NDM, OXA, IMP y VIM (J.C. Hormazábal, comunicación personal, 2017).

Si bien K. pneumoniae es la principal especie productora de KPC, también se ha determinado su presencia en otras especies de la familia Enterobacteriaceae, como K. oxytoca, Salmonella enterica, E. coli, Proteus spp., Citrobacter freundii, Enterobacter spp., Raoultella ornithinolytica, Morganella morganii, Pantoea spp., Providencia spp. y S. marcescens ${ }^{23-29}$. Adicionalmente, se ha determinado la presencia de esta enzima en bacilos gramnegativos no fermentadores como A. baumannii, P. aeruginosa y Flavobacterium odoratum ${ }^{17,30-31}$. Esta enzima presenta actividad contra un amplio espectro de antimicrobianos $\beta$-lactámicos que incluye penicilinas, cefalosporinas, cefamicinas, aztreonam y carbapenémi$\cos ^{8}$. Su actividad in vitro es pobremente inhibida por ácido clavulánico y tazobactam y presenta una elevada inhibición por ácido fenilborónico ${ }^{32}$.

\begin{tabular}{|c|c|c|c|c|c|}
\hline $\begin{array}{l}\text { Grupo funcional/ } \\
\text { Clase Ambler }\end{array}$ & $\begin{array}{l}\text { Tipos de } \\
\text { enzimas }\end{array}$ & Espectro de actividad & Inhibidores & Distribución geográfica & Loaclización molecular \\
\hline $2 f / A$ & $\begin{array}{c}\text { KPC: } \mathrm{KPC}-2 \\
\text { a } 24\end{array}$ & $\begin{array}{l}\text { Penicilinas, cefalosporinas, } \\
\text { cefamicinas, carbapenémicos }\end{array}$ & $\begin{array}{l}\text { Clavulanato, tazobactam, } \\
\text { sulbactam, ácido borónico }\end{array}$ & $\begin{array}{l}\text { Estados Unidos, Grecia, Italia, } \\
\text { Israel, China, Brasil, Colombia, } \\
\text { Argentina, Chile }\end{array}$ & $\begin{array}{l}\text { Tn4401, plásmidos de tipo } \\
\text { IncFII, CC258 }\end{array}$ \\
\hline $3 / B$ & $\begin{array}{l}\text { MLBs: NDM-1, } \\
\text { IMP, VIM }\end{array}$ & $\begin{array}{l}\text { Penicilinas, cefalosporinas, } \\
\text { cefamicinas, carbapenémicos }\end{array}$ & EDTA, ácido dipicolínico & $\begin{array}{l}\text { Japón (IMP), Taiwán (IMP), India } \\
\text { (NDM), Grecia (VIM), Chile (NDM) }\end{array}$ & $\begin{array}{l}\text { Plásmidos de tipo IncA/C, } \\
\mathrm{N}(\mathrm{NDM}) \text {, integrón clase I } \\
\text { (VIM, IMP) }\end{array}$ \\
\hline $2 \mathrm{~d} / \mathrm{D}$ & $\begin{array}{c}\text { OXA-48, 181 } \\
204,232\end{array}$ & $\begin{array}{l}\text { Penicilinas, inhibidor de } \\
\beta \text {-lactamasas, carbapenémicos } \\
\text { (débil) }\end{array}$ & $\mathrm{NaCl}$ & $\begin{array}{l}\text { Turquía, África del Norte, Europa } \\
\text { (España, Bélgica), Chile }\end{array}$ & $\begin{array}{l}\text { Tn1999, plásmidos de tipo } \\
\text { IncL/M }\end{array}$ \\
\hline
\end{tabular}

Adaptado de Pitout et al. ${ }^{8}$ 
En la actualidad se han descrito 24 alelos del gen $b l a_{\mathrm{KPC}}$, los que difieren entre sí por 1- 3 aminoácidos ${ }^{33-34}$. Estas variantes de KPC han sido clasificadas en número secuencial desde $b l a_{\mathrm{KPC}-1}$ a $b l a_{\mathrm{KPC}-24}$ (https://www.lahey. org/studies/ 6 de junio de 2017), no obstante, la secuencia aminoacídica de la variante de KPC-1 y KPC-2 presentan $100 \%$ de identidad; por lo tanto, corresponden a la misma enzima $^{35}$. Además de la diferente secuencia aminoacídica, las variantes de KPC difieren en sus propiedades cinéticas, por lo que pueden presentar diferencias en la eficiencia hidrolítica de los carbapenémicos y de otros antimicrobianos $\beta$-lactámicos ${ }^{36}$. Las variantes KPC-2 y KPC-3 son las más comunes entre los aislados clínicos $\mathrm{y}$ son responsables de los brotes epidémicos ${ }^{15}$, siendo KPC-2 la enzima predominante en el mundo, con brotes informados en E.U.A., Europa y China, mientras que KPC-3 ha sido principalmente detectada en E.U.A., Israel y América Latina ${ }^{37}$.

\section{Epidemiología de KPC}

Las enterobacterias productoras de carbapenemasas no sólo han sido aisladas en el ambiente hospitalario, sino que también desde ambientes no hospitalarios. En el año 2012, Poirel y cols. ${ }^{38}$, informan por primera vez el aislamiento de E. coli productora de KPC desde aguas costeras en Portugal. Posteriormente, se informó el aislamiento de este tipo de cepas en agua de pozo, riveras, lagos, plantas de tratamiento de aguas residuales y producción animal ${ }^{23,39-41}$. Por lo tanto, la presencia de bacterias productoras de carbapenemasas en el ambiente es alarmante por el potencial riesgo de diseminación de genes de resistencia. Además, con estos hallazgos se refuerza la idea que el medio ambiente puede participar en la selección y diseminación de genes de resistencia ${ }^{39}$.

La rápida diseminación mundial del gen $b l a_{\mathrm{KPC}}$ ha sido atribuido a una combinación de factores sociales y microbiológicos: viajes internacionales, transmisión paciente-paciente de micro-organismos productores de KPC y transferencia horizontal de genes ${ }^{4,42-46}$. Esta rápida diseminación, también varía geográficamente, determinándose así regiones que informan pocos aislados productores de KPC (Australia y África) y áreas donde KPC es considerada endémica (E.U.A., Puerto Rico, Colombia, Grecia, Israel y China $)^{15}$. Entre los factores microbiológicos, se ha determinado que el transposón Tn4401 contribuye tanto a la diseminación geográfica de KPC como a la transferencia interespecie ${ }^{43,47}$. Sin embargo, la rápida diseminación de los genes $b l a_{\mathrm{KPC}}$ en múltiples especies también ha sido como consecuencia de su presencia en una amplia variedad de plásmidos que varían en tamaño, naturaleza y estructura ${ }^{48-49}$. Otro factor involucrado es la asociación de la enzima KPC con clones de alto riesgo, los que tienen una distribución global, mayor capacidad de colonización, diseminación y persistencia en una variedad de nichos ${ }^{43,50}$.

La enzima KPC está presente en más de 100 secuenciotipos (ST) diferentes, pero la pandemia de KPC es debido, principalmente, a la propagación de miembros del complejo clonal (CC) 258 con el ST258 y las variantes ST11, ST340 y ST512 ${ }^{51}$. Los miembros de este linaje son típicamente resistentes a todos los antimicrobianos, excepto colistín, tigeciclina y gentamicina ${ }^{52}$. Sin embargo, se ha informado la emergencia y diseminación de otros ST como ST307, el que está apareciendo en diferentes lugares del mundo y podría convertirse en un nuevo clon pandémico ${ }^{53}$.

En el CC258, el ST258 es el clon predominante y ha sido identificado en aproximadamente $70 \%$ de las cepas de K. pneumoniae productoras de KPC. El clon ST11 es predominante en $\mathrm{China}^{54}$, el ST258 en Europa, Medio Oriente, E.U.A. y Latinoamérica ${ }^{15,34,55}$; por otra parte, el ST437 también ha sido identificado en hospitales de Latinoamérica ${ }^{56}$. Además, el clon ST258, está compuesto por dos linajes genéticos, denominados clado I y clado II. El clado I está asociado con KPC-2 y el clado II con KPC3. La divergencia genética entre estos dos clados radica en una región de $215 \mathrm{~Kb}$ que incluye genes involucrados en la síntesis de la cápsula de polisacárido $(\mathrm{cps})^{57}$. Esta región de divergencia sería un hot spot para eventos de recombinación y, según DeLeo y cols. ${ }^{58}$, contribuiría en la exitosa diseminación del clon ST258.

Por otra parte, entre los primeros aislados productores de KPC detectados en Chile, el ISP identificó el ST116159. Este ST corresponde a un nuevo ST y, además, es considerado autóctono, ya que sólo ha sido identificado en este país.

\section{Entorno genético del gen $b l a_{\mathrm{KPC}}$}

La enzima KPC se encuentra codificada por el gen $b l a_{\mathrm{KPC}}$, localizado en el transposón Tn4401, derivado de Tn3, o en elementos genéticos similares a Tn4401, el que a su vez se encuentra portado, principalmente, en plásmidos aunque también ha sido informado en el cromosoma ${ }^{12}$. Tn4401 tiene un tamaño aproximado de $10 \mathrm{~Kb}$ y está delimitado por dos secuencias de repeticiones invertidas imperfectas de $39 \mathrm{pb}$ y se encuentra flanqueado por un sitio blanco de duplicación de $5 \mathrm{pb}$, lo que indicaría un reciente evento de transposición. Además, contiene los genes de la transposasa $(\operatorname{tnp} A)$, la resolvasa $(\operatorname{tnp} R)$ y dos secuencias de inserción, ISKpn6 e ISKpn7, río arriba del gen $b l a_{\mathrm{KPC}}{ }^{60}$. El transposón Tn4401 es la principal plataforma de diseminación de $b l a_{\mathrm{KPC}} \mathrm{y}$ la frecuencia de transposición es de 4,4 x 10-6 célula receptora $^{8}$.

Se han descrito ocho isoformas de Tn4401 (a-h, con dos isoformas diferentes de Tn4401d) (Figura 1). Las 
isoformas a, c, d y e difieren entre sí por deleciones río arriba de bla $_{\mathrm{KPC}}$, de $99 \mathrm{pb}, 215 \mathrm{pb}, 68 \mathrm{pb}$ y $255 \mathrm{pb}$, respectivamente, en comparación con la isoforma $b$ que no presenta deleción ${ }^{61-64}$. Adicionalmente, Chen y cols..$^{65}$, describieron una nueva isoforma del Tn4401 la que designaron también como Tn4401d. Esta isoforma se caracteriza por tener una deleción de $5,3 \mathrm{~Kb}$ que incluye parte del gen $b l a_{\mathrm{KPC}}$; por lo tanto, la enzima no sería funcional. Posteriormente, Kendall y cols. ${ }^{66}$, informaron que KPC-4 aislada desde E. cloacae y $S$. marcescens está codificada en la variante Tn4401f que contiene el gen tnpA truncado y tnpR, ISKpn 7 y Tn4401 IR-L están ausentes. Recientemente, Cheruvanky y cols. ${ }^{67}$, describieron una nueva isoforma de Tn4401, designada como Tn4401h, que se caracteriza por tener una deleción de 188 pb entre los genes $i s t B$ y $b l a_{\mathrm{KPC}}$, y fue identificada en $K$. pneumoniae y E. cloacae.

Actualmente, se han descrito nuevas plataformas genéticas donde se encuentra el gen $b l a_{\mathrm{KPC}}$, las cuales se diferencian de Tn4401 debido a otras deleciones e inserciones de otros genes y a la adición de otras secuencias de inserción ${ }^{17,36,68-70}$. En las nuevas plataformas descritas, los principales cambios ocurren en la región río arriba del gen $b l a_{\mathrm{KPC}}$, lo que sugiere que esta región es variable ${ }^{60}$. Shen y cols. ${ }^{70}$, caracterizaron una nueva plataforma genética del gen $b l a_{\mathrm{KPC}}$ en cepas de enterobacterias aisladas en China. Así, al secuenciar el plásmido pK048, determinaron una región de $2.070 \mathrm{pb}$ idéntica a Tn4401 que incluye el gen $b l a_{\mathrm{KPC}-2} \mathrm{y}$ un fragmento parcial de la secuencia de inserción ISK pn6. Esta plataforma fue generada por la integración de un transposón del tipo Tn3 río arriba del gen $b l a_{\mathrm{KPC}-2}$ y la inserción de otros genes río abajo de la secuencia de inserción ISKpn6, causando la pérdida parcial de esta secuencia. Posteriormente, esta plataforma ha sido descrita con frecuencia en dicho país y, además, variantes

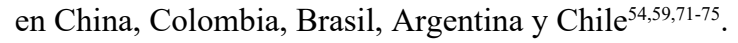

En el año 2011, Gómez y cols. ${ }^{74}$, determinaron una variante de la plataforma descrita en China, a la que denominaron variante 1a Argentina (Figura 2). En esta plataforma se encontró la inserción de un transposón compuesto, río arriba de tnp $A$, que provocó la deleción parcial de este último y, además, la inserción de un fragmento del gen $b l a_{\mathrm{TEM}-1}$ truncado $(671 \mathrm{pb})$ entre ISKpn 8 y $b l a_{\mathrm{KPC}}$. Cabe destacar que Barría-Loaiza y cols. ${ }^{59}$, determinaron tanto la presencia de la plataforma Tn4401a como de la variante 1a en cepas de enterobacterias productoras de KPC-2 aisladas en hospitales chilenos, siendo la variante 1a la más común (11/17 cepas). Por otra parte, Ageevets y cols. ${ }^{68}$, describieron un elemento genético móvil de $17.003 \mathrm{pb}$ que albergaría el gen $b l a_{\mathrm{KPC}-2}$. Este elemento se encuentra flanqueado por repeticiones invertidas de $38 \mathrm{pb}$ del Tn3, que incluye el gen tnpA-Tn3, el operón de resistencia a macrólidos $(m p h A-m r x-m p h R)$ y un fragmento del gen $b l a_{\mathrm{TEM}-1}$.

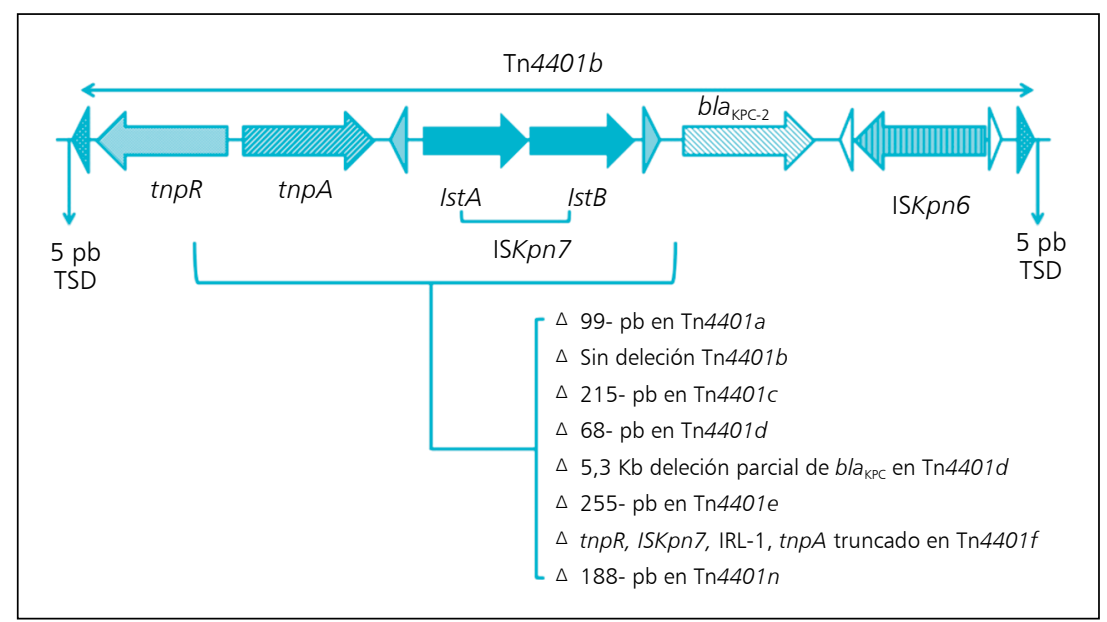

Figura 1. Estructura del transposón Tn4401 y sus isoformas a-f. IRL: repetición invertida izquierda; tnpR: gen que codifica la resolvasa; tnpA: gen que codifica la transposasa; ist $A$ e ist $B$ : genes que conforman la secuencia de inserción ISKpn7; ISKpn6: secuencia de inserción; TSD: sitio de duplicaciones diana. Los triángulos negros representan las secuencias repetidas invertidas. Adaptado de Nordmann y cols. ${ }^{34}$.

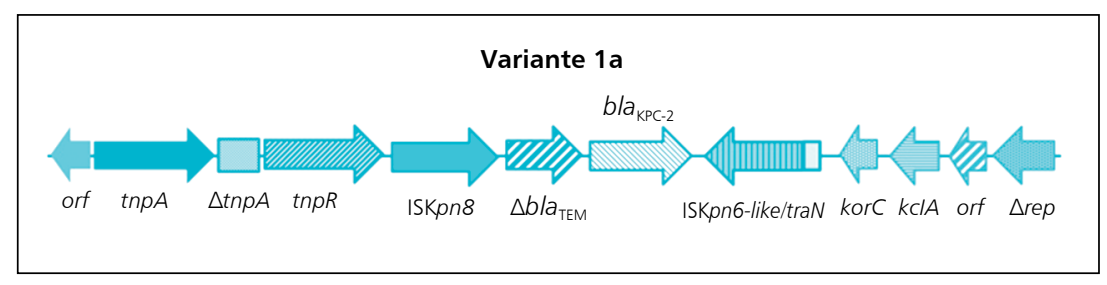

Figura 2. Estructura de la variante 1a Argentina de la plataforma genética 1a descrita en China, $\triangle \operatorname{tnp} A-T n 3$ : gen que codifica la transposasa delecionado; $\triangle b / a_{\mathrm{TEM}-1}$ : gen que codifica la $\beta$-lactamasa TEM-1 delecionado. Adaptado de Barría-Loaiza y cols. ${ }^{59}$.

El Tn4401 ha sido detectado en aislados de diferentes orígenes geográficos, distintos $\mathrm{ST}$, variadas especies de enterobacterias y también en $P$. aeruginos $a^{48}$. Una característica importante es su capacidad de insertarse en diferentes plásmidos de bacterias gramnegativas y ha sido identificado en plásmidos conjugativos y no conjugativos movilizables. Los plásmidos que albergan el gen $b l a_{\mathrm{KPC}}$ suelen estar asociados con otros determinantes genéticos, los cuales confieren resistencia a otros antimicrobianos como fluoroquinolonas, aminoglucósidos y cotrimoxa$\mathrm{zol}^{76}$. En relación a los tipos de plásmidos que contienen el gen $b l a_{\mathrm{KPC}}$ en aislados del ST258, se ha determinado que pertenecen a diferente grupo de incompatibilidad IncF (replicones FIIK1, FIIK2 y FIA), Incl2, IncX, IncA/C, IncR, IncL/M y ColE1, siendo el grupo de incompatibilidad IncF, con el replicón FIIK, el predominante ${ }^{69}$. Estos plásmidos tienen una amplia distribución geográfica que incluye Canadá, Polonia, E.U.A., Italia, Israel, Brasil y Noruega $^{56,69}$. Adicionalmente, en la caracterización de los primeros aislados de enterobacterias productoras de KPC en Chile, se identificó que el gen $b l a_{\mathrm{KPC}-2}$ esta asociado a 
variantes de plásmidos del grupo IncF, lo que concuerda con lo descrito en los países anteriormente mencionados ${ }^{77}$.

\section{Factores asociados a los diferentes niveles de CIM de carbapenémicos}

Las cepas productoras de KPC exhiben un amplio rango de concentración inhibitoria mínima (CIM) de carbapenémicos, entre el rango de susceptible (CIM $<1 \mu \mathrm{g} / \mathrm{mL})$ a altamente resistente $(\mathrm{CIM}>256 \mu \mathrm{g} / \mathrm{mL})^{34,78}$. Esta variación en la CIM de carbapenémicos sobre cepas productoras de KPC hace difícil su detección por sistemas automatizados, lo cual conduce a fracaso en la detección oportuna $^{79}$.

Se ha determinado que la producción de KPC más la pérdida de porina puede conducir a una CIM elevada de carbapenémicos ${ }^{80}$. Landman y cols. ${ }^{81}$, informaron que en aislados de $K$. pneumoniae productores de KPC, la CIM de ertapenem se incrementó con la disminución de la expresión del gen ompK36 y además, los resultados del grado de expresión del gen $b l a_{\mathrm{KPC}-2}$ exhibidos en algunas cepas resistentes a carbapenémicos no fueron consistentes con la CIM de carbapenémicos ${ }^{82}$. Con estos resultados, se sugirió que la enzima KPC por sí sola podría no ser suficiente para conferir resistencia a carbapenémicos ${ }^{62,83}$ y se propuso la participación de otros mecanismos adicionales ${ }^{81}$. Así, junto a la participación de las porinas como mecanismo adicional, se determinó que las bombas de expulsión AcrAB participan en la resistencia a $\beta$-lactámicos en cepas de K. pneumoniae ${ }^{84,85}$; por lo tanto, podrían estar involucradas en las variaciones de la CIM de carbapenémicos en aislados productores de KPC.

Por otra parte, otro factor asociado al amplio rango de CIM de carbapenémicos corresponde a las deleciones río arriba del gen $b l a_{\mathrm{KPC}}$, específicamente entre el gen ist $B$ y $b l a_{\mathrm{KPC}}{ }^{47}$. En la isoforma Tn4401a, la que presenta una deleción de $99 \mathrm{pb}$, adyacente a la región promotora del gen $b l a_{\mathrm{KPC}}$, produce una secuencia promotora -35 diferente a la isoforma $\operatorname{Tn} 4401 \mathrm{~b}^{49}$ (sin deleción), lo que impacta en el grado de expresión del gen $b l a_{\mathrm{KPC}} \mathrm{y}$, por lo tanto, en el grado de resistencia a carbapenémicos ${ }^{61,62}$. Además, los aislados productores de KPC pueden contener múltiple número de copias del gen $b l a_{\mathrm{KPC}}$, como consecuencia de que el gen puede estar localizado dentro del mismo plásmido en múltiples copias o puede estar en múltiples plásmidos $^{49}$. Por lo tanto, el número de copias también afectaría el nivel de producción de KPC y, por ende, puede contribuir a modificar el grado de resistencia a los carbapenémicos y el aumento de la velocidad de hidrólisis de carbapenémicos ${ }^{62,81}$. No obstante, Roth y cols. ${ }^{86}$, señalaron que el número de copias y los grados de expresión del gen $b l a_{\mathrm{KPC}}$, no explicarían la variación de los patrones de susceptibilidad de los aislados clínicos y de las cepas transformadas con el gen $b l a_{\mathrm{KPC}-2}$, ya que al comparar cepas con un alto y bajo número de copias del gen $b l a_{\mathrm{KPC}}$, los valores de CIM no presentaron diferencias. Por otra parte, se ha identificado que el gen $b l a_{\mathrm{KPC}}$ puede ser expresado desde diferentes promotores (P1, P2 y $\mathrm{P} 3$ ), pero que los promotores $\mathrm{P} 1$ y $\mathrm{P} 2$ contribuyen a la expresión del gen $b l a_{\mathrm{KPC}} \mathrm{y}$ podría conducir a variaciones en la CIM de carbapenémicos y en diferentes grados de expresión de $b l a_{\mathrm{KPC}}{ }^{61}$. Sin embargo, otros autores, señalan que la presencia de diferentes promotores no es suficiente para explicar el elevado grado de resistencia a carbapenémicos ${ }^{86}$. Con estos resultados se establece que mecanismos adicionales a la producción de KPC (bombas expulsión, expresión de porinas y/o producción de BLEE/ AmpC) estarían contribuyendo a la variabilidad en los grados de resistencia a carbapenémicos.

\section{Implicancias clínicas de KPC}

Las infecciones causadas por bacterias productoras de KPC no muestran especificidad por un órgano o tejido; sin embargo, factores de riesgo como hospitalización prolongada, estadía en unidades de cuidados intensivos, pacientes inmunodeprimidos, dispositivos invasores, terapia antimicrobiana previa, recepción de trasplantes y ventilación mecánica, han sido asociados a la adquisición o infección con bacterias productoras de $\mathrm{KPC}^{34,87-88}$

Aunque se ha relacionado una mayor mortalidad en pacientes infectados con cepas productoras de KPC que con cepas que no producen esta enzima, no se han identificado factores de virulencia específicos asociados con bacterias productoras de $\mathrm{KPC}^{34,89-90}$. Por ejemplo, un estudio empleando Galleria mellonella como modelo indica que los aislados de K. pneumoniae productores de KPC fueron menos virulentos que los aislados no productores ${ }^{91}$. Por otra parte, utilizando el modelo de Caenorhabditis elegans se determinó que el gen $b l a_{\mathrm{KPC}}$ por sí solo no está asociado con un incremento en la virulencia ${ }^{90}$, lo que también es informado por Chiang y cols. ${ }^{92}$, quienes determinaron que las diferentes variantes de KPC no están asociadas con un incremento en la virulencia de K. pneumoniae. Sin embargo, recientemente se ha informado que el genoma de K. pneumoniae ST307 codifica características genéticas que podrían proporcionar una ventaja en la adaptación al entorno hospitalario y al hospedador humano. El análisis de la secuencia reveló nuevos factores de virulencia localizados en un plásmido, incluyendo un cluster de genes para la síntesis de glucógeno, lo que es considerado como una de las posibles respuestas adaptativas a la supervivencia y crecimiento de la cepa por periodos prolongados en entornos fuera del hospedador ${ }^{53}$.

Las infecciones por enterobacterias resistentes a carbapenémicos (ERC) se asocian a una elevada mortalidad. Por ejemplo, se informan valores de $25-75 \%$, en el caso de infecciones ${ }^{93}$ y de incluso $50 \%$ en pacientes con 
bacteriemias $^{94}$. Este problema es debido, principalmente, a las escasas opciones terapéuticas de estas infecciones, limitadas a colistín y tigeciclina.

Colistín es un lipopéptido cíclico, perteneciente al grupo de las polimixinas que, al menos desde un punto de vista teórico, es un antimicrobiano bactericida cuyo sitio blanco es el lípido A del lipopolisacárido ${ }^{95}$. Lamentablemente, aún existe mucho por conocer en relación a su dosificación, farmacocinética, metodologías a emplear para el estudio de susceptibilidad y puntos de corte, entre otros ${ }^{96}$. Su principal limitación es la nefrotoxicidad que, en promedio, alcanza a 30\% ${ }^{95}$. Además, existe una creciente descripción de resistencia por mecanismos cromosomales y también plasmídicos, que pueden limitar el éxito y aplicación de esta polimixina ${ }^{96}$, considerada la última opción terapéutica sobre bacilos gramnegativos extremadamente resistentes a drogas (XDR).

Tigeciclina es un antimicrobiano bacteriostático, perteneciente al grupo de las glicilciclinas. A pesar de su amplio espectro antibacteriano, su uso se encuentra aprobado sólo para el tratamiento de infecciones complicadas de piel y tejidos blandos, neumonía adquirida en la comunidad y de infecciones intrabdominales complicadas. Su utilización en bacteriemias es controversial por la baja concentración plasmática alcanzada tras administrar dosis habituales, y su baja excreción urinaria limita su uso en infecciones del tracto urinario (ITU) ${ }^{97}$.

Una importante cantidad de trabajos observacionales, la mayoría retrospectivos y no aleatorizados, sugieren que existe beneficio, en términos de supervivencia, al combinar antimicrobianos para el tratamiento de infecciones por $\mathrm{ERC}^{98}$, sobre todo con esquemas que contienen un carbapenémico, lo que necesariamente debe ser confirmado en ensayos clínicos controlados que se encuentran actualmente en marcha. Finalmente, existen antimicrobianos no disponibles en Chile y que han demostrado eficacia en el tratamiento de infecciones por ERC. Por un lado, se encuentra fosfomicina, un antiguo inhibidor de la síntesis de peptidoglicano (en la fase intracelular) que ha demostrado tener actividad sobre ERC, especialmente $K$. pneumoniae productora de $\mathrm{KPC}^{99}$. El uso de este antimicrobiano se encuentra limitado por la FDA para el tratamiento vía oral de ITU no complicadas ${ }^{100}$. Por otro lado, los diaza-biciclo-octanos corresponden a una nueva generación de inhibidores de $\beta$-lactamasas no $\beta$-lactámicos, capaces de inactivar muchas de las $\beta$-lactamasas clase C y A (incluyendo KPC), pero no las de clase B. Sus representantes son avibactam y relebactam, estando ya el primero aprobado por la FDA en asociación con ceftazidima (ceftazidima/avibactam) para el tratamiento de ITU complicada e infecciones intraabdominales complicadas. Esta combinación ha demostrado eficacia in vitro contra cepas de enterobacterias no susceptibles a carbapenémicos; no obstante, los estudios pivotales que determinaron su aprobación no incluían $\mathrm{ERC}^{101}$. Como era de esperar, ya hay descripción de resistencia a dicha asociación $^{102,103}$.

\section{Conclusiones}

La principal enterobacteria productora de la enzima carbapenemasa KPC es $K$. pneumoniae y la producción de esta enzima es el mecanismo más importante asociado con la resistencia a los antimicrobianos carbapenémicos. Las cepas productoras de KPC pueden presentar un grado de resistencia heterogéneo a los carbapenémicos, dificultando su detección, y consiguientemente la pesquisa, resultando en alta mortalidad en las infecciones provocadas por estos aislados debido al retraso de un tratamiento oportuno. Se ha determinado que el diferente grado de expresión del gen $b l a_{\mathrm{KPC}}$, la alteración en la expresión de las porinas y la participación de bombas de expulsión asociada a la síntesis de BLEE y/o AmpC podrían estar produciendo las variaciones en los grados de resistencia a carbapenémicos. Por lo tanto, es necesario estudiar los mecanismos moleculares involucrados en la resistencia a carbapenémicos en las cepas productoras de KPC, ya que la información generada permitirá mejorar los métodos de detección de KPC y, por ende, prevenir los fracasos en el tratamiento terapéutico.

\section{Resumen}

En la actualidad, la diseminación de enterobacterias productoras de carbapenemasas se considera un grave problema en clínica debido al fracaso en el tratamiento de las infecciones que ellas producen. Entre las carbapenemasas, la enzima KPC se ha diseminado mundialmente y ha sido identificada en las principales especies de enterobacterias relacionadas con infecciones asociadas a la atención en salud, con claro predominio de Klebsiella pneumoniae a nivel mundial. El gen $b l a_{\mathrm{KPC}}$ es transportado, principalmente, por el transposón Tn4401, detectado en diversas especies de enterobacterias con distintos secuencio-tipo (ST) y diferente origen geográfico. Adicionalmente, se han descrito nuevas plataformas genéticas que se distinguen del Tn4401 original debido a inserciones y deleciones de otros genes. Los plásmidos que albergan el gen $b l a_{\mathrm{KPC}}$ pueden ser del tipo conjugativo y no conjugativo movilizable, y además contener otros determinantes genéticos de resistencia. Las cepas productoras de KPC pueden presentar diversos niveles de resistencia a los carbapenémicos, debido a la participación de mecanismos adicionales como diferente grado de expresión de porinas y bombas de expulsión asociados con la producción de $\beta$-lactamasas de espectro extendido y/o AmpC. Sin embargo, las carbapenemasas, con KPC como la enzima más frecuente, otorgan grados de resistencia más elevados. 


\section{Referencias bibliográficas}

1.- $\quad$ Yang Y J, Wu P, Livermore D M. Biochemical characterization of a $\beta$-lactamase that hydrolyzes penems and carbapenems from two Serratia marcescens isolates. Antimicrob Agents Chemother 1990; 34: 755-8.

2.- Rasmussen B A, Bush K, Keeney D, Yang Y, Hare R, O'Gara C, et al. Characterization of IMI-1 $\beta$-lactamase, a class A carbapenemhydrolyzing enzyme from Enterobacter cloacae. Antimicrob Agents Chemother 1996; 40: 2080-6.

3.- Bush K, Jacoby G A, Medeiros A A. A functional classification scheme for betalactamases and its correlation with molecular structure. Antimicrob Agents Chemother 1995; 39: 1211-33.

4.- Queenan A M, Bush K. Carbapenemases: the versatile beta-lactamases. Clin Microbiol Rev 2007; 20: 440-58.

5.- Gupta N, Limbago B M, Patel J B, Kallen A J. Carbapenem- resistant Enterobacteriaceae: epidemiology and prevention. Clin Infect Dis 2011; 53: 60-7.

6.- Evans B A, Amyes S G. OXA betalactamases. Clin Microbiol Rev 2014, 27: 241-63.

7.- Nordmann P, Dortet L, Poirel L. Carbapenem resistance in Enterobacteriaceae: here is the storm! Trends Mol Med 2012; 18: 263-72.

8.- Pitout J D, Nordmann P, Poirel L. Carbapenemase-producing Klebsiella pneumoniae, a key pathogen set for global nosocomial dominance. Antimicrob Agents Chemother 2015; 59: 5873-84. 10.1128/ AAC.01019-15

9.- World Health Organization. Antimicrobial resistance: global report on surveillance 2014 . Geneva, Switzerland: WHO; 2014.

10.- World Health Organization. www. who. int/ mediacentre/ news/ releases/2017 / bacteriaantibiotics-needed/en/WHO Junio 2017.

11.- Yigit H, Queenan A M, Anderson G J, Domenech-Sanchez A, Biddle J W, Steward $\mathrm{CD}$, et al. Novel carbapenem-hydrolyzing $\beta$-lactamase KPC-1 from a carbapenemresistant strain of Klebsiella pneumoniae. Antimicrob Agents Chemother 2001; 45: 115161.

12.- Escandón-Vargas K, Reyes S, Gutiérrez $\mathrm{S}$, Villegas M V. The epidemiology of carbapenemases in Latin America and the Caribbean. Expert Rev Anti Infect Ther 2017; 15: 277-97.

13.- Bakour S, Sahli F, Touati A, Rolain J M. Emergence of KPC-producing Klebsiella pneumoniae ST512 isolated from cerebrospinal fluid of a child in Algeria. New Microbes New Infect 2015; 3: 34-6.

14.- Chang L W, Buising K L, Jeremiah C J, Cronin K, Poy Lorenzo Y S, Howden B P, et al. Managing a nosocomial outbreak of carbapenem-resistant Klebsiella pneumoniae: an early Australian hospital experience. Intern Med J 2015; 45: 1037-43.

15.- Chen L, Anderson D, Paterson D. Overview of the epidemiology and the threat of Klebsiella pneumonia carbapenemases (KPC) resistance. Infect Drug Resist 2012; 5: 133-41.

16.- Villegas M V, Lolans K, Correa A, Suárez C J, López J A, Vallejo M, et al. The Colombian Nosocomial Resistance Study Group. First detection of the plasmid mediated class A carbapenemase KPC-2 in clinical isolates of Klebsiella pneumoniae from South America. Antimicrob Agents Chemother 2006; 50: 28802.

17.- Cuzon G, Naas T, Villegas M V, Correa A, Quinn J P, Nordmann P. Wide dissemination of Pseudomonas aeruginosa producing lactamase bla $_{\mathrm{KPC}-2}$ gene in Colombia. Antimicrob Agents Chemother 2011; 55: 5350-3.

18.- López J A, Correa A, Navon-Venezia S, Correa A L, Torres J A, Briceño D F, et al. Intercontinental spread from Israel to Colombia of a KPC-3 producing Klebsiella pneumoniae strain. Clin Microbiol Infect 2011; 17: 52-6.

19.- Pasteran F, Faccone D, Gómez S, De Bunder S, Spinelli F, Rapoport M, et al. Detection of an international multiresistant clone belonging to sequence type 654 involved in the dissemination of KPC-producing Pseudomonas aeruginosa in Argentina. J Antimicrob Chemother 2012; 67: 1291-3.

20.- Peirano G, Seki L M, Val Passos V L, Pinto M C, Guerra L R, Asensi M D. Carbapenemhydrolysing beta-lactamase KPC-2 in Klebsiella pneumoniae isolated in Rio de Janeiro, Brazil. J Antimicrob Chemother 2009; 63: 265-8.

21.- Cifuentes M, García P, San Martín P, Silva F, Zúñiga J, Reyes $S$, et al. Primer caso de detección de $b l a_{\mathrm{KPC}}$ en Chile: desde Italia a un hospital público de Santiago. Rev Chilena Infectol 2012; 29: 224-8.

22.- ISP 2015. Instituto de Salud Pública. Boletín Instituto de Salud Pública, Vol 5, No 4, 2015

23.- Sun P, Bi Z, Nilsson M, Zheng B, Berglund $\mathrm{B}$, Stålsby Lundborg $\mathrm{C}$, et al. Occurrence of $b l a_{\mathrm{KPC}-2}, b l a_{\mathrm{CTX}-\mathrm{M}}$, and $m c r-1$ in Enterobacteriaceae from well water in rural China. Antimicrob Agents Chemother 2017; 24. doi: 10.1128/AAC.02569-16

24.- Aires C A, Almeida A C, Vilela M A, MoraisJunior M A, Morais M M. Selection of KPC-2producing Providencia stuartii during treatment for septicemia. Diag Microbiol Infect Dis 2016; 84: 95-6.

25.- Saldanha Ribeiro P C, Souza Monteiro A, Garcia Marquez S, Gomes Monteiro S, Monteiro-Neto V, Melo Coqueiro M M, et al. Phenotypic and molecular detection of the $b l a_{\mathrm{KPC}}$ gene in clinical isolates from inpatients at hospitals in São Luis, MA, Brazil. BMC Infect Dis 2016; 16: 737.

26.- Shi D S, Wang W P, Kuai S G, Shao H F, Huang M. Identification of $b l a_{\mathrm{KPC}-2}$ on different plasmids of three Morganella morganii isolates. Eur J Clin Microbiol Infect Dis 2012; 31: 797 803.

27.- Bratu S, Brooks S, Burney S, Kochar S, Gupta J, Landman D, et al. Detection and spread of Escherichia coli possessing the plasmid-borne carbapenemase KPC-2 in Brooklyn, New York. Clin Infect Dis 2007; 44: 972-5.

28.- Miriagou V, Tzouvelekis L S, Rossiter S, Tzelepi E, Angulo F J, Whichard J. Imipenem resistance in a Salmonella clinical strain due to plasmid-mediated class A carbapenemase KPC-2. Antimicrob Agents Chemother 2003; 47: 1297-1300.

29.- Yigit H, Queenan A M, Rasheed J K, Biddle J W, Domenech-Sanchez A, Ablerti S, et al. Carbapenem-resistant strains of Klebsiella oxytoca harboring carbapenem-hydrolyzing $\beta$ - lactamase KPC-2. Antimicrob Agents Chemother 2003; 47: 3881-9.

30.- Kuai S, Huang L, Pei H, Chen Y, Liu $\mathrm{J}$. Imipenem resistance due to class A carbapenemase KPC-2 in Flavobacterium odoratum isolate. J Med Microbiol 2011; 60: 1408-9.

31.- Robledo I E, Aquino E, Sante M, Santana J, Otero D, Leo C, et al. Detection of KPC in Acinetobacter spp. in Puerto Rico. Antimicrob Agents Chemother 2010; 54: 1354-7.

32.- Nordmann P, Poirel L. Strategies for identification of carbapenemase-producing Enterobacteriaceae. J Antimicrob Chemother 2013; 68: 487-9.

33.- Doumith M, Findlay J, Hirani H, Hopkins K L, Livermore D M, Dodgson A, et al. Major role of $\mathrm{pKpQIL-like} \mathrm{plasmids} \mathrm{in} \mathrm{the} \mathrm{early}$ dissemination of KPC-type carbapenemases in the UK. J Antimicrob Chemother 2017; 11. doi: $10.1093 / \mathrm{jac} / \mathrm{dkx} 141$.

34.- Nordmann P, Cuzon G, Naas T. The real threat of Klebsiella pneumoniae carbapenemase producing bacteria. Lancet Infect Dis 2009; 9: 228-36.

35.- Yigit H, Queenan A M, Anderson G J, Domenech-Sanchez A, Biddle J W, Steward C D, et al. Author's correction- Novel carbapenem- hydrolyzing beta-lactamase, KPC-1, from a carbapenem-resistant strain of Klebsiella pneumoniae. Antimicrob Agents Chemother 2008; 52: 809.

36.- Wolter D J, Kurpiel P M, Woodford N, Palepou M F, Goering R V, Hanson N D. Phenotypic and enzymatic comparative analysis of the novel KPC variant KPC-5 and its evolutionary variants, KPC-2 and KPC-4. Antimicrob. Agents Chemother 2009; 53: 557-62.

37.- Lee C R, Lee J H, Park K S, Kim Y B, Jeong B C, Lee S H. Global dissemination 
of carbapenemase-producing Klebsiella pneumoniae: Epidemiology, genetic context, treatment options, and detection methods. Front Microbiol 2016; 7: 895.

38.- Poirel L, Barbosa-Vasconcelos A, Simoes R R, Da Costa P M, Liu W, Nordmann P. Environmental KPC-producing Escherichia coli isolates in Portugal. Antimicrob Agents Chemother 2012; 56: 1662-3.

39.- Piedra-Carrasco N, Fàbrega A, Calero- Cáceres W, Cornejo-Sánchez T, Brown-Jaque M, Mir-Cros A, et al. Carbapenemase- producing Enterobacteriaceae recovered from a Spanish river ecosystem. PLoS ONE 2017; 12: e0175246. https://doi.org/10.1371/journal. pone. 0175246 .

40.- Yang F, Mao D, Zhou H, Luo Y. Prevalence and fate of carbapenemase genes in a wastewater treatment plant in Northern China. PLoS ONE 2016; 11: e0156383.

41.- Woodford N, Wareham D W, Guerra B, Teale C. Carbapenemase-producing Enterobacteriaceae and non-Enterobacteriaceae from animals and the environment: an emerging public health risk of our own making? J Antimicrob Chemother 2014; 69: 287-91.

42.- Goodman K E, Simner P J, Tamma P D, Milstone A M. Infection control implications of heterogeneous resistance mechanism in carbapenem-resistant Enterobacteriaceae (CRE). Expert Rev Anti Infect Ther 2016; 14: 1-14.

43.- Nordmann P, Poirel L. The difficult-to-control spread of carbapenemase producers among Enterobacteriaceae worldwide. Clin Microbiol Infect 2014; 20: 821-30.

44.- Bush K, Fisher J F. Epidemiological expansion, structural studies, and clinical challenges of new $\beta$-lactamases from gram-negative bacteria. Annu Rev Microbiol 2011; 65: 455-78.

45.- Sidjabat H E, Silveira F P, Potoski B A, AbuElmagd K M, Adams-Haduch J M, Paterson D L, et al. Interspecies spread of Klebsiella pneumoniae carbapenemase gene in a single patient. Clin Infect Dis 2009; 49: 1736-8.

46.- Naas T, Nordmann P, Vedel G, Poyart C. Plasmid-mediated carbapenem- hydrolyzing beta-lactamase KPC in a Klebsiella pneumoniae isolate from France. Antimicrob Agents Chemother 2005; 49: 4423-4.

47.- Naas T, Cuzon G, Villegas M V, Lartigue M F, Quinn J P, Nordmann P. Genetic structures at the origin of acquisition of the beta-lactamase $b l a_{\mathrm{KPC}}$ gene. Antimicrob Agents Chemother 2008; 52: 1257-63.

48.- Cuzon G, Naas T, Truong H, Villegas M V, Wisell K T, Carmeli Y, et al. Worldwide diversity of Klebsiella pneumoniae that produce beta-lactamase $b l a_{\mathrm{KPC}-2}$ gene. Emerg Infect Dis 2010; 16:1349-56.

49.- Gootz T D, Lescoe M K, Dib-Hajj F, Dougherty B A, He W, Della-Latta P, et al. Genetic organization of transposase regions surrounding $b l a_{\mathrm{KPC}}$ carbapenemase genes on plasmids from Klebsiella strains isolated in a New York City hospital. Antimicrob Agents Chemother 2009; 53: 1998-2004.

50.- Baquero F, Tedim A P, Coque T M. Antibiotic resistance shaping multi-level population biology of bacteria. Front Microbiol 2013; 4:15

51.- Peirano G, Bradford P, Kazmierczak K, Chen L, Kreiswirth B, Pitou J. The importance of clonal complex 258 and IncFK2-like plasmids among a global collection of Klebsiella pneumoniae with bla $_{\mathrm{KPC}}$. Antimicrob Agents Chemother 2017; doi: 10.1128/AAC.02610-16.

52.- Livermore D. Current epidemiological and growing resistance of Gram negative pathogens. Korean J Intern Med 2012; 27: 12842.

53.- Villa L, Feudi C, Fortini D, Brisse S, Passet $\mathrm{V}$, Bonura $\mathrm{C}$, et al. Diversity, virulence, and antimicrobial resistance of the KPC producing Klebsiella pneumoniae ST307 clone. Microbial genomics 2017; DOI 10.1099/mgen.0.000110.

54.- Qi Y, Wei Z, Ji S, Du X, Shen P, Yu Y. ST11, the dominant clone of KPC-producing Klebsiella pneumoniae in China. J Antimicrob Chemother 2011; 66: 307-12.

55.- Castanheira M, Costello A J, Deshpande L M, Jones R N. Expansion of clonal complex 258 KPC-2-producing Klebsiella pneumonia in Latin American hospitals: report of the SENTRY Antimicrobial Surveillance Program. Antimicrob Agents Chemother 2012; 56: 16689.

56.- Andrade L N, Curiao T, Ferreira J C, Longo J M, Climaco E C, Martinez R, et al. Dissemination of $b l a_{\mathrm{KPC}-2}$ by the spread of Klebsiella pneumoniae clonal complex 258 clones (ST258, ST11, ST437) and plasmids (IncFII, IncN, IncL/M) among Enterobacteriaceae species in Brazil. Antimicrob Agents Chemother 2011; 55: 3579 -83 .

57.- Mathers A J, Stoesser N, Sheppard A E, Pankhurst L, Giess A, Yeh A J, et al. Klebsiella pneumoniae carbapenemase (KPC)-producing K. pneumoniae at a single institution: Insights into endemicity from whole-genome sequencing. Antimicrob Agents Chemother 2015; 59: 1656-63.

58.- DeLeo F R, Chen L, Porcella S F, Martens C A, Kobayashi S D, Porter A R, et al. Molecular dissection of the evolution of carbapenemresistant multilocus sequence type 258 Klebsiella pneumoniae. Proc Natl Acad Sci USA 2014; 111: 4988- 93.

59.- Barría-Loaiza C, Pincheira A, Quezada M, Vera A, Valenzuela P, Domínguez M, et al. Molecular typing and genetic environment of the bla $_{\mathrm{KPC}}$ gene in Chilean isolates of Klebsiella pneumoniae. J Glob Antimicrob Resist 2016; 4: 28-34.
60.- Cuzon G, Naas T, Nordmann P. Functional characterization of $\operatorname{Tn} 4401$, a $\operatorname{Tn} 3$ - based transposon involved in $b l a_{\mathrm{KPC}}$ gene mobilization. Antimicrob Agents Chemother 2011; 55: 5370-3.

61.- Naas T, Cuzon G, Truong HV, Nordmann P. Role of ISKpn7 and deletions in $b l a_{\mathrm{KPC}}$ gene expression. Antimicrob Agents Chemother 2012; 56: 4753-9.

62.- Kitchel B, Rasheed J K, Endimiani A, Hujer A M, Anderson K F, Bonomo R A, et al. Genetic factors associated with elevated carbapenem resistance in KPC-producing Klebsiella pneumoniae. Antimicrob Agents Chemother 2010; 54: 4201-7.

63.- Naas T, Cuzon G, Villegas M V, Lartigue M F, Quinn J P, Nordmann P. Genetic structures at the origin of acquisition of the beta-lactamase $b l a_{\mathrm{KPC}}$ gene. Antimicrob Agents Chemother 2008; 52:1257-63.

64.- Kitchel B, Rasheed J K, Patel J B, Srinivasan A, Navon-Venezia S, Carmeli Y, et al. Molecular epidemiology of KPC- producing Klebsiella pneumoniae isolates in the United States: clonal expansion of multilocus sequence type 258. Antimicrob Agents Chemother 2009; 53: 3365-70.

65.- Chen L, Chavda K D, Mediavilla J R, Jacobs M R, Levi M H, Bonomo R A, et al. Partial excision of $b l a_{\mathrm{KPC}}$ from Tn4401 in carbapenemresistant Klebsiella pneumoniae. Antimicrob Agents Chemother 2012; 56: 1635-8.

66.- Kendall A B, Van Schooneveld T, Thapa I, Bastola D, Williams L, Safranek T, et al. KPC-4 is encoded within a truncated Tn4401 in an IncL/ M plasmids, pNe1280, isolated from Enterobacter cloacae and Serratia marcescens. Antimicrob Agents Chemother 2013; 57: 37-41.

67.- Cheruvanky A, Stoesser N, Sheppard A E, Crook D W, Hoffman P S, Weddle E, et al. Enhanced Klebsiella pneumoniae carbapenemase (KPC) expression from a novel Tn4401 deletion. Antimicrob Agents Chemother 2017. doi: 10.1128/AAC.00025-17.

68.- Ageevets V, Sopova J, Lazareva I, Malakhova M, Ilina E, Kostryukova E, et al. Genetic environment of the $b l a_{\mathrm{KPC}-2}$ gene in a Klebsiella pneumoniae isolate that may have been imported to Russia from Southeast Asia. Antimicrob Agents Chemother 2017; 61: e01856-16. https://doi.org/10.1128/ AAC.01856-16.

69.- Chen L, Chavda K D, Melano R G, Jacobs M $\mathrm{R}$, Levi M H, Bonomo R A, et al. Complete sequence of a bla(KPC-2)-harboring $\operatorname{IncFII(K1)}$ plasmid from a Klebsiella pneumoniae sequence type 258 strain. Antimicrob Agents Chemother 2013; 57: 1542-5.

70.- Shen P, Wei Z, Jiang Y, Du X, Ji S, Yu Y, et al. Novel genetic environment of the carbapenem- 
hydrolyzing beta-lactamase KPC-2 among Enterobacteriaceae in China. Antimicrob Agents Chemother 2009; 53: 4333-8.

71.- Anchordoqui M S, De Belder D, Lucero C, Rapoport M, Faccone D, Rodriguez A, et al. In vivo horizontal dissemination of the $b l a_{\mathrm{KPC}-2}$ gene carried on diverse genetic platforms among clinical isolates of Enterobacteriaceae. Glob Antimicrob Resist 2015; 3: 210-3.

72.- Naas T, Bonnin R A, Cuzon G, Villegas M $\mathrm{V}$, Nordmann P. Complete sequence of two KPC-harbouring plasmids from Pseudomonas aeruginosa. J Antimicrob Chemother 2013; 68: 1757-62.

73.- Ribeiro V B, Zavascki A P, Nodari C S, Sandri A M, Silva M P, Campos J C, et al. Detection of $b l a_{\mathrm{KPC}-2}$ in a carbapenem-resistant Kluyvera georgiana. J Antimicrob Chemother 2012; 67: 2776-7.

74.- Gómez S A, Pasteran F G, Faccone D, Tijet N, Rapoport M, Lucero C, et al. Clonal dissemination of Klebsiella pneumoniae ST258 harbouring KPC-2 in Argentina. Clin Microbiol Infect 2011; 17: 1520-4.

75.- Li G, Wei Q, Wang Y, Du X, Zhao Y, Jiang X. Novel genetic environment of the plasmidmediated KPC-3 gene detected in Escherichia coli and Citrobacter freundii isolates from China. Eur J Clin Microbiol Infect Dis 2011; 30: 575-80.

76.- Endimiani A, Carias L L, Hujer A M, Bethel C R, Hujer K M, Perez F, et al. Presence of plasmid-mediated quinolone resistance in Klebsiella pneumoniae isolates possessing $b l a_{\mathrm{KPC}}$ in the United States. Antimicrob Agents Chemother 2008; 52: 2680-2.

77.- Geisse J. Plásmidos relacionados a $b l a_{\mathrm{KPC}}$ en cepas de enterobacterias chilenas productoras de carbapenemasas. Tesis para optar al grado de Magíster en Ciencias mención Microbiología. Universidad de Concepción, Chile, 2017.

78.- Anderson K F, Lonsway D R, Rasheed J $\mathrm{K}$, Biddle J, Jensen B, McDougal L K, et al. Evaluation of methods to identify the Klebsiella pneumoniae carbapenemase in Enterobacteriaceae. J Clin Microbiol 2007; 45 : 2723-5.

79.- Adams-Sapper S, Nolen S, Donzelli G F, Lal M, Chen K, Justo da Silva L H, et al. Rapid induction of high-level carbapenem resistance in heteroresistant KPC-producing Klebsiella pneumoniae. Antimicrob Agents Chemother 2015; 59: 3281-9.

80.- Zhang R, Yang L, Cai J C, Zhou H W, Chen G X. High-level carbapenem resistance in a Citrobacter freundii clinical isolate is due to a combination of KPC-2 production and decreased porin expression. J Med Microbiol 2008; 57: 332-7.

81.- Landman D, Bratu S, Quale J. Contribution of OmpK36 to carbapenem susceptibility in
KPC-producing Klebsiella pneumoniae. J Med Microbiol 2009; 58: 1303-8.

82.- Seecoomar G D, Marmol B C, Kwon D H. Promoter deletions of Klebsiella pneumoniae carbapenemase (KPC)-encoding genes $\left(b a_{\mathrm{KPC}-2}\right)$ and efflux pump (AcrAB) on beta-lactam susceptibility in KPC-producing Enterobacteriaceae. FEMS Microbiol Lett 2013; 348: 120-6.

83.- Roth A, Thomson K S, Lister P D, Hanson N. Production of KPC-2 alone does not always result in $\beta$-lactam MICs representing resistance in gram-negative pathogens. J Clin Microbiol 2012; 50: 4183-4.

84.- Pages J M, Lavigne J P, Leflon-Guibout V, Marcon E, Bert F, Noussair L, et al. Efllux pump, the masked side of $\beta$-Lactam resistance in Klebsiella pneumoniae clinical isolates. PLoS ONE 2009; 4: 1-9.

85.- Hasdemir U O, Chevalier J, Nordmann P, Pagès J M. Detection and prevalence of active drug efflux mechanism in various multidrugresistant Klebsiella pneumoniae strains from Turkey. J Clin Microbiol 2004; 42: 2701-6.

86.- Roth A, Kurpiel P, Lister P, Hanson N. Gen $b l a_{\mathrm{KPC}}$ RNA expression correlates with two transcriptional star sites but not always with gene copy number in four genera of Gramnegative pathogens. Antimicrobial Agents Chemother 2011; 55: 3936-8.

87.- Marchaim D, Navon-Venzia S, Schwaber M J, Carmeli Y. Isolation of imipenem-resistant Enterobacter species; emergence of KPCcarbapenemase, molecular characterization, epidemiology and outcomes. Antimicrob Agents Chemother 2008; 52: 1413-8.

88.- Bratu S, Landman D, Haag R, Recco R, Eramo A, Alam M, et al. Rapid spread of carbapenem-resistant Klebsiella pneumoniae in New York City. Arch Intern Med 2005; 165 : 1430-5.

89.- Hennequin C, Robin F. Correlation between antimicrobial resistance and virulence in Klebsiella pneumoniae. Eur J Clin Microbiol Infect Dis 2016; 35: 333-41.

90.- Lavigne J P, Cuzon G, Combescure C, Bourg G, Sotto A, Nordmann P. Virulence of Klebsiella pneumoniae isolates harboring $b l a_{\mathrm{KPC}-2}$ carbapenemase gene in a Caenorhabditis elegans model. PLoS ONE 2013; 8(7):e67847. https://doi.org/10.1371/journal.pone.0067847.

91.- McLaughlin M M, Advincula M R, Malczynski M, Barajas G, Qi C, Scheetz M H. Quantifying the clinical virulence of Klebsiella pneumoniae producing carbapenemase Klebsiella pneumoniae with a Galleria mellonella model and a pilot study to translate to patient outcomes. BMC Infect Dis 2014; 14: 31; http:// dx.doi.org/10.1186/1471-2334-14-31.

92.- Chiang T T, Yang Y S, Yeh K M, Chiu S K, Wang N C, Lin T Y, et al. Quantification and comparison of virulence and characteristics of different variants of carbapenemase-producing Klebsiella pneumoniae clinical isolates from Taiwan and the United States. J Microbiol Immunol Infect 2016; 49: 83-90.

93.- Tzouvelekis L S, Markogiannakis A, Psichogiou M, Tassios T T, Daikos G L. Carbapenemases in Klebsiella pneumoniae and other Enterobacteriaceae: an evolving crisis of global dimensions. Clin Microbiol Rev 2012; 25: 682-707.

94.- Patel G, Huprikar S, Factor S H, Jenkins S G, Calfee D P. Outcomes of carbapenem-resistant Klebsiella pneumoniae infection and the impact of antimicrobial and adjunctive therapies. Infect Control Hosp Epidemiol 2008; 29: 1099-106.

95.- Aguayo A, Mella S, Riedel G, Bello H, Domínguez M, González-Rocha G. Colistín en la era post-antibiótica. Rev Chilena Infectol 2016; 166-76.

96.- Poirel L, Jayol A, Nordmann P. Polymyxins: Antibacterial activity, susceptibility testing, and resistance mechanisms encoded by plasmids or chromosomes. Clin Microbiol Rev 2017; 30: 557-96.

97.- Stein G E, Babinchak T. Tigecycline: an update. Diagn Microbiol Infect Dis 2013; 75: 331-6.

98.- Tumbarello M, Trecarichi E M, De Rosa F G, Gianella M, Giacobbe D R, Bassetti M, et al. Infections caused by KPC-producing Klebsiella pneumoniae: differences in therapy and mortality in a multicentre study. J Antimicrob Chemother 2015; 70: 2133-43.

99.- Kaase M, Szabados F, Anders A, Gatermann S G. Fosfomycin susceptibility in carbapenemresistant Enterobacteriaceae from Germany. J Clin Microbiol 2014; 52: 1893-7.

100.-Bowers D R, Huang V. Emerging issues and treatment strategies in carbapenem-resistant Enterobacteriaceae (CRE). Curr Infect Dis Rep 2016; 18: 48-55.

101.-Pérez F, El Chakhtoura N G, Papp-Wallace K M, Wilson B M, Bonomo R A. Treatment options for infections caused by carbapenemresistant Enterobacteriaceae: can we apply "precision medicine" to antimicrobial chemotherapy? Expert Opin Pharmacother 2016; 17: 761-81.

102.-Humphries R M, Hemarajata P. Resistance to ceftazidime-avibactam in Klebsiella pneumoniae due to porin mutations and the increased expression of KPC-3. Antimicrob Agents Chemother 2017; 61. doi: 10.1128/ AAC.00537-17.

103.-Haidar G, Clancy C J, Shields R K, Hao B, Cheng S, Nguyen M H. Mutations in $b a_{\mathrm{KPC}-3}$ that confer ceftazidime-avibactam resistance encode novel KPC-3 variants that function as extended-spectrum $\beta$-lactamases. Antimicrob Agents Chemother 2017; 61. doi: 10.1128/ AAC.02534-16. 